OPEN ACCESS

Edited by:

Yang Zhang,

Dalian University of Technology, China

Reviewed by:

Ying Ma,

North China University of Water Conservancy and Electric Power,

China

Hai-Bin Huang,

Hebei University of Technology, China

*Correspondence:

Xin-Chi Yan

skywalker@jiangnan.edu.cn

Specialty section:

This article was submitted to Interdisciplinary Physics,

a section of the journal

Frontiers in Physics

Received: 25 May 2021

Accepted: 04 June 2021

Published: 23 June 2021

Citation:

Cheng H, Yan X-C and Fu L (2021)

Distant Relative Genetic

Algorithm-Based Structural

Reliability Optimization.

Front. Phys. 9:714381.

doi: 10.3389/fphy.2021.714381

\section{Distant Relative Genetic Algorithm-Based Structural Reliability Optimization}

\author{
Hu Cheng, Xin-Chi Yan* and Li Fu \\ School of Environment and Civil Engineering, Jiangnan University, Wuxi, China
}

In this study, safety margin explicit equation has been established using random variables (i.e., the engineering conditions, structure parameters, structural strength, and external load), and the genetic algorithm (GA)-based structural reliability optimization design has been addressed subsequently. Though the conventional adaptive GA can change automatically with fitness, it is still not unsatisfactory in sufficiently improving the algorithm convergence speed, especially for complex structures. This article presents an improved adaptive technology termed as the distant relative genetic algorithm (DRGA), in which the distant relative pointer and immunity operators can effectively improve the search performance of the GA. In early evolution, by means of cross controlling and avoiding pairing between individuals with the same genes, the methodology prevents the isogenic individuals expanding locally. Besides, the revised algorithm is able to jump out of the local optimal solution, thus ensuring the realization of a fast global convergence. An example based on wing box structure optimization has been demonstrated using the improved method, and the calculation results show that this strategy makes the GA more effective in dealing with the constraint optimization issues.

Keywords: reliability-based structural optimization, genetic algorithm, wing box structure, distant relative pointer, immunity operator

\section{INTRODUCTION}

With the rapid development of large and complex structures, more and more attention has been paid to the structural safety, such as the structural control and health monitoring $[1,2]$, the long-term performance deterioration $[3,4]$, and the structural optimization $[5,6]$, where the reliability-based structural optimization (RBSO) with multiple stochastic variables is always a difficult problem [7]. The weight and material cost of a structure can be effectively reduced by selecting the structural reliability index as the constraint of the optimization problem and adopting the element crosssectional area, length, strength, and external load as the stochastic variables. Meanwhile, the structure strength, stiffness, and resistance to vibration and stability can also be improved. An optimized RBSO design, which is beneficial to make structures meet the requirements of economy and safety, has essential application value.

In the past few decades, much attention has been paid to the standard genetic algorithm (SGA) [8, 9]. However, since actual structures are usually large-scale and complicated, shortcomings such as premature convergence and poor computational efficiency frequently appear when the SGA is applied to the RBSO [10]. The above limitations make many researchers and engineers doubt about whether the genetic algorithm (GA) can be applied to the optimization design for actual structures [11]. 
The selection, crossover, and mutation operations of the GA are random, which ensure the evolution of population. The individual degradation phenomenon, however, appears due to the arrangement of the algorithm. Crossover and mutation probabilities are the two genetic parameters that are key to the performance of the GA. Srinivas and Patnaik [12] proposed that the adaptive genetic algorithm (AGA) can change automatically with fitness. But, the conventional AGA methodology is still unsatisfactory in sufficiently improving the algorithm convergence speed.

To deal with such aforementioned drawbacks, an improved adaptive technology, termed as the distant relative genetic algorithm (DRGA), is proposed in this study. In the following, the element safety margin function and system failure analysis are introduced, focusing on the optimization problem of an ideal elastic-plastic beam-slab structure system. Then the improved adaptive technology is presented, followed by the detailed operation process. After that, a wing box structure is taken as the numerical example for the validation of the proposed method, and finally, conclusions are drawn.

\section{ELEMENT SAFETY MARGIN FUNCTION AND SYSTEM FAILURE ANALYSIS}

An ideal elastic-plastic beam-slab structure system is focused on for the optimization problem. The considered material parameters are mainly the strength and elastic modulus. As for the geometrical parameter, it includes the slab (i.e., the length, width, and thickness) and the beam (the area, length, and moment of inertia).

The safety margin of the slab element can be expressed as follows:

$$
Z_{B}=\sigma_{a}-\sigma_{e o}
$$

where $\sigma_{a}$ is the maximum stress allowed; $\sigma_{e o}$ is the equivalent stress at the center of the slab element, which can be determined by the following equation:

$$
\sigma_{e o}=\left(\sigma_{x o}^{2}+\sigma_{y o}^{2}-\sigma_{x o} \sigma_{y o}+3 \tau_{x y o}^{2}\right)^{\frac{1}{2}},
$$

where $\sigma_{x o}$ and $\sigma_{y o}$ are the normal stresses in the local coordinate system, and $\tau_{x y o}$ is the shear stress in the local coordinate system [13].

Let $\overline{\boldsymbol{\delta}}=\left(\begin{array}{llllllll}\bar{u}_{1} & \bar{v}_{1} & \bar{u}_{2} & \bar{v}_{2} & \bar{u}_{3} & \bar{v}_{3} & \bar{u}_{4} & \bar{v}_{4}\end{array}\right)^{T}$ be the nodal displacement vector in the local coordinate system, then

$$
\left\{\begin{array}{c}
\sigma_{x o} \\
\sigma_{y o} \\
\tau_{x y o}
\end{array}\right\}=\Lambda\left[\begin{array}{cccccccc}
-b & -\mu a & b & -\mu a & b & \mu a & -b & \mu a \\
-\mu b & -a & \mu a & -a & \mu a & a & -\mu a & a \\
-\alpha a & -\mu b & -\mu a & \mu b & \mu a & \mu b & \mu a & -\mu b
\end{array}\right]\left\{\begin{array}{c}
\bar{u}_{1} \\
\bar{v}_{1} \\
\bar{u}_{2} \\
\bar{v}_{2} \\
\bar{u}_{3} \\
\bar{v}_{3} \\
\bar{u}_{4} \\
\bar{v}_{4}
\end{array}\right\}
$$

where $\Lambda=E / 2 a b\left(1-\mu^{2}\right), \quad \alpha=(1-\mu) / 2, \quad E$ is the elasticity modulus, $\mu$ is Poisson ratio, and $a$ and $b$ are the side lengths of the slab.
It can be seen that the safety margin of the slab element $Z_{B}$ depends on the nodal displacement and the parameters of the rectangular element (i.e., length, width, and elasticity modulus).

When any left end section of the beam element fails, the safety margin of the beam can be determined by adopting the criterion of linear approximation as follows:

$$
Z_{2 q-1}=R_{q}-\mathbf{C}_{2 q-1} \overline{\mathbf{F}}
$$

where $R_{q}$ is the resistance of the straight beam $q$ with a constant cross section, $\mathbf{C}_{2 q-1}$ is the matrix of coefficient related to the parameters of beam, and $\overline{\mathbf{F}}$ is the vector of node loads applied to the beam [13]. Then the safety margin equation turns as follows:

$$
\begin{aligned}
Z_{2 q-1}= & \sigma_{y} \bar{w}_{z q}-\left[\frac{\bar{w}_{z q}}{A_{q}} \operatorname{sg}\left(\bar{N}_{2 q-1}\right) \bar{N}_{2 q-1}+\frac{\sqrt{3}}{2} \frac{\bar{w}_{z q}}{\bar{A}_{y q}} \operatorname{sg}\left(\bar{N}_{y, 2 q-1}\right) \bar{N}_{y, 2 q-1}\right. \\
& +\frac{\sqrt{3}}{2} \frac{\bar{w}_{z q}}{\bar{A}_{z q}} \operatorname{sg}\left(\bar{N}_{z, 2 q-1}\right) \bar{N}_{z, 2 q-1}+\frac{\bar{w}_{z q}}{\bar{w}_{x q}} \operatorname{sg}\left(\bar{M}_{x, 2 q-1}\right) \bar{M}_{x, 2 q-1} \\
& \left.+\frac{\bar{w}_{z q}}{\bar{w}_{y q}} \operatorname{sg}\left(\bar{M}_{y, 2 q-1}\right) \bar{M}_{y, 2 q-1}+\operatorname{sg}\left(\bar{M}_{z, 2 q-1}\right) \bar{M}_{z, 2 q-1}\right]=0
\end{aligned}
$$

where $\sigma_{y}$ is the yield stress of elements, $A_{q}$ is the sectional area of beam $q, \bar{A}_{y q}$ and $\bar{A}_{z q}$ are the shearing areas of beam $q$ in the directions of $\bar{y}$ and $\bar{z}$, respectively, $\bar{w}_{x q}$ is the plastic torsional coefficient on $\bar{x}$ axis, and $\bar{w}_{z q}$ and $\bar{w}_{y q}$ are the plastic section modulus which corresponds to the moments $\bar{M}_{z}$ and $\bar{M}_{y}$ of beam $q$, respectively.

In a similar way, the safety margins can also be deduced when the right end section of the beam fails to $\bar{z}$ axis and both ends fail to $\bar{y}$ axis.

When the components of the system fail, the stress redistribution will occur to the structure. The reduced stiffness matrix of system members must be reconsidered. Once the number of failed components reaches to a particular number $p_{q}$, the system stiffness matrix will be singular as follows:

$$
\left|\mathbf{K}^{p_{q}}\right|=0,
$$

where $\mathbf{K}^{p_{q}}$ is the reduced system stiffness matrix with $p_{q}$ failed components. Accordingly, the structural system fails.

Structure fails means that the structure turns into machinery. In order to calculate the system reliability index, all failure modes or integrated failure paths should be identified, but it is impossible and unnecessary in the analysis for complex structures. Alternatively, the typical failure modes can be adopted. The branch-and-bound method [14] is regarded as a proper approach to identify the typical failure modes. By boundary function operations, the number of subsystems can be reduced for structural reliability analysis. When all typical failure modes are identified, the system reliability index can be figured out by using the probability network evaluation technique [15]:

$$
P_{f_{s}}=1-\prod_{i=1}^{G}\left(1-P_{f i}\right),
$$

where $P_{f_{s}}$ is the failure probability of the structural system, $P_{f i}$ is the failure probability of the typical failure mode, and $G$ is the number of typical failure modes. 


\section{ADAPTIVE STRATEGY WITH DISTANT RELATIVE POINTER}

\section{Adaptive Strategy}

The typical adaptive strategy makes the crossover probability $\left(p_{c}\right)$ and mutation probability $\left(p_{m}\right)$ change according to the individual fitness. When the individual fitness becomes accordant, it will make $p_{c}$ and $p_{m}$ increase. Otherwise, $p_{c}$ and $p_{m}$ will decrease when the individual fitness are diverse. In the meantime, lower values of $p_{c}$ and $p_{m}$ will be given to protect individuals whose fitness is larger than the average population fitness, and vice versa. The self-manipulated change of $p_{c}$ and $p_{m}$ can be described as follows [12]:

$$
\begin{aligned}
& p_{c}=\left\{\begin{array}{ll}
\frac{k_{1}\left(f_{\max }-f^{*}\right)}{f_{\max }-\bar{f},} & f^{*} \geq \bar{f} \\
k_{2}, & f^{*}<\bar{f}
\end{array},\right. \\
& p_{m}=\left\{\begin{array}{ll}
\frac{k_{3}\left(f_{\max }-f\right)}{f_{\max }-\bar{f}}, & f \geq \bar{f} \\
k_{4}, & f<\bar{f}
\end{array},\right.
\end{aligned}
$$

where $f_{\max }$ is the maximum population fitness, $\bar{f}$ is the average population fitness, $f^{*}$ is the larger crossover individual fitness, and $f$ is the mutation individual fitness. The parameters (i.e., $k_{1}$, $k_{2}, k_{3}$, and $k_{4}$ ) range from 0 to 1 .

The sufficient conditions of a global optimization ability are as follows: 1) a local search ability and 2) the ability of suddenly jumping from one local optimization to the next better one. However, the local search ability of the conventional AGA is inefficient in the early evolution because the optimal individual in the population almost does not change. Meanwhile, in the later evolution process, the optimal individual does not have the capability to jump out of the local optimal solution, leading to local convergence rather than global convergence eventually.

According to Eqs. 8, 9, when the population tends to be consistent, the AGA tends to increase $p_{c}$ and $p_{m}$. However, in fact the consistence may be caused by any super individual existing in the population. Selecting operation may cause these individuals to overwhelm repeatedly in the new generations of population. An important consequence of this is a destroyed diversity of the population and consequently a premature convergence. The AGA alone cannot accommodate this phenomenon that once an overwhelming individual exists in the population, increasing $p_{c}$ cannot generate new models nor make the calculation jump out of the local optimal solution. On the other hand, increasing the variability (e.g., the $p_{m}$ ) of the AGA may cause the algorithm to degrade into random search with fewer poor individuals depending on variability. Accordingly, convergence in this case is inefficient and unstable.

\section{Distant Relative Pointer}

In order to enhance the global optimization ability of the GA, individual expansion phenomenon must be mitigated, which has to be done at the early cross operation. Setting distant relative pointer is essential, and the detailed calculation process is performed as follows.

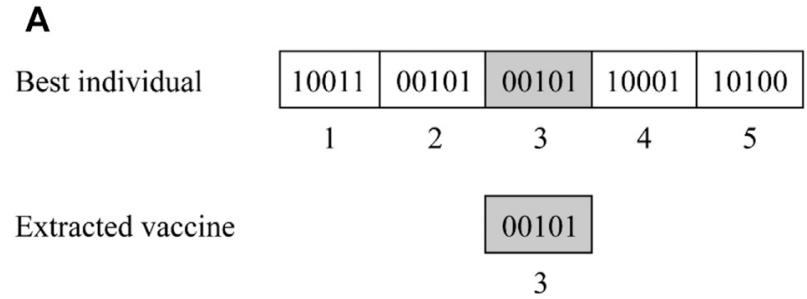

B

\begin{tabular}{|c|c|c|c|c|c|}
\hline \multirow[t]{2}{*}{ Before } & 00010 & 10101 & 10101 & 11001 & 10101 \\
\hline & 1 & 2 & 3 & 4 & 5 \\
\hline \multirow[t]{2}{*}{ After } & 00010 & 10101 & 00101 & 11001 & 10101 \\
\hline & 1 & 2 & 3 & 4 & 5 \\
\hline
\end{tabular}

Extract vaccine

FIGURE 1 | Schematic diagram for the extraction and inoculation processes.

Assume that the population is denoted by $P(t)=\left\{x_{i}\right\}$ when calculating the $t$ th generation. If one individual is selected twice in the $t+1$ th generation, its location numbers will be remembered, and a distant relative pointer will be allocated to each of them. After that, if one individual is selected to be crossed, it must be paired with a different one who has no identical relative pointer. Then any other marked individual will be operated as an immunity operator, and the $p_{c}$ and $p_{m}$ of other individuals of this population are still calculated based on Eqs. 8, 9.

The proposed distant relative pointer can effectively avoid any individual to dominate in the population. Meanwhile, the best individuals will not be damaged. This strategy has the capability to generate a new model, and the overall operation is simple. The algorithm also assures a diversity of the population, and at the same time, it is able to avoid local optimization and ensure the global convergence of the GA.

\section{Immunity Operator}

In order to prevent an individual from degenerating in basic operations via the GA, the immunity operator is adopted, which consists of a vaccine inoculation and immunity selection.

\section{Vaccine Inoculation}

A vaccine is a basic indicator of the specific question involved. In the RBSO, a vaccine can be a calculation result or a theoretical deduction. Figure 1 shows a scheme of the extraction and inoculation processes. Assume the $t$ th generation is being calculated, the corresponding population is $P(t)=\left\{x_{i}\right\}$, and the random $m(m \leq n)$ individuals can be used as the vaccine for inoculation. The $m$ is calculated using the following normalized equation:

$$
m=a \times n,
$$




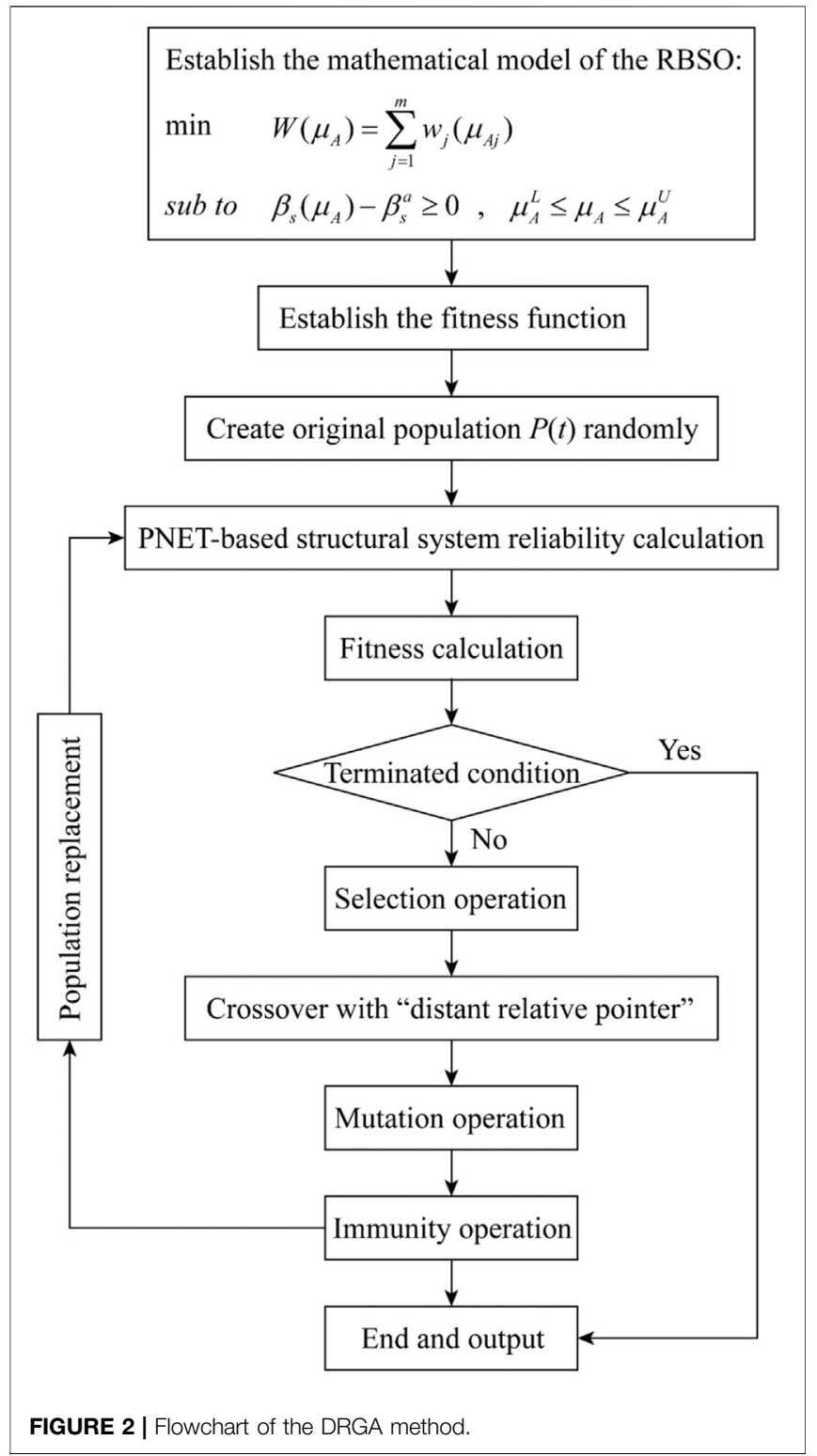

where $a=0.05 \sim 0.1$ is the inoculate probability, and $n$ is the population size.

\section{Immunity Selection}

The individuals that have been inoculated with the vaccine will be inspected subsequently. If their fitness is worse than that of the last generation, they will be replaced by their "fathers." If their fitness is better than that of the last generation, it means the inoculation is successful. The appeared new optimal individual will be selected in the new generation.

\section{OPERATION PROCESS OF THE DRGA METHOD}

Based on the above research, this article presents a distant relative genetic algorithm (DRGA) method to solve the RBSO question.

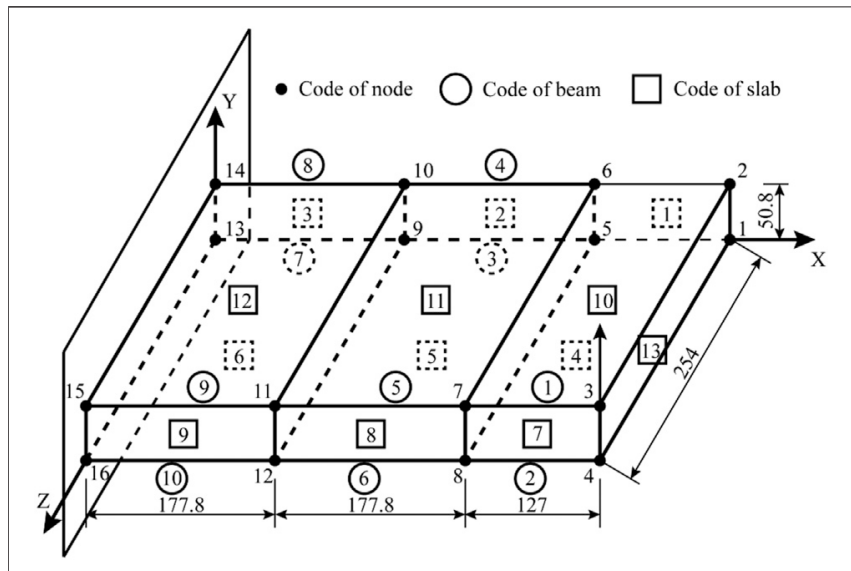

FIGURE 3 | Wing box structure $(\mathrm{mm})$

Figure 2 shows the flowchart of the computer program, and the significant procedures are performed as follows.

\section{Stage I: Establish the Mathematical Model of the RBSO}

In this step, constraints function must be expressed definitely. For an RBSO question, it is usually expressed as $\beta_{s} \geq \beta_{s}^{a}$, where $\beta_{s}$ is the structural system reliability, and $\beta_{s}^{a}$ is boundary value of $\beta_{s}$.

\section{Stage II: Establish the GA Fitness Function for the Optimization Question}

Lagrange multiplier method [16] is adopted to deduce the fitness function, which can be expressed as follows:

$$
F=C-W-\frac{1}{2 r}\left\{\max \left[0, \lambda+r\left(\beta_{s}-\beta_{s}^{a}\right)\right]^{2}-\lambda^{2}\right\},
$$

where $F$ is the fitness value, $C$ is a large constant, which can be adopted as 1.25 times the mass of the structural system when all design variables equal the maximum values, $W$ is the structural mass, $\lambda$ is the Lagrange multiplier and $\lambda_{k+1}=\max \left\{0, \lambda_{k}+r\left(\beta_{s}-\beta_{s}^{a}\right)\right\}$, and $r$ is the penalty factor, which is greater than zero.

\section{Stage III: Calculate the Structural System Reliability}

After deducing the safety margin explicit equation, the branchand-bound method is adopted to search the main failure modes, and the probabilistic network evaluation technique (PNET) is adopted to calculate the system reliability index.

\section{Stage IV: Create the Immunity Operator and Perform the GA Optimization Based on Distant Relative Pointer}

Except for the first three primary operators, the immunity operator and distant relative pointer are necessary to be 
TABLE 1 | Optimized cross-sectional areas of beams.

\begin{tabular}{|c|c|c|c|c|c|c|c|c|c|c|}
\hline $\begin{array}{l}\text { Serial } \\
\text { number }\end{array}$ & 1 & 2 & 3 & 4 & 5 & 6 & 7 & 8 & 9 & 10 \\
\hline Cross-sectional area $\left(\mathrm{cm}^{2}\right)$ & 9.03 & 8.91 & 6.28 & 5.62 & 4.65 & 6.32 & 5.03 & 6.62 & 8.98 & 9.71 \\
\hline
\end{tabular}

\begin{tabular}{|c|c|c|c|}
\hline Serial number & Thickness (cm) & Serial number & Thickness (cm) \\
\hline 1 & 0.37 & 8 & 0.47 \\
\hline 2 & 0.37 & 9 & 0.51 \\
\hline 3 & 0.32 & 10 & 0.48 \\
\hline 4 & 0.31 & 11 & 0.34 \\
\hline 5 & 0.28 & 12 & 0.32 \\
\hline 6 & 0.26 & 13 & 0.41 \\
\hline 7 & 0.49 & - & - \\
\hline
\end{tabular}

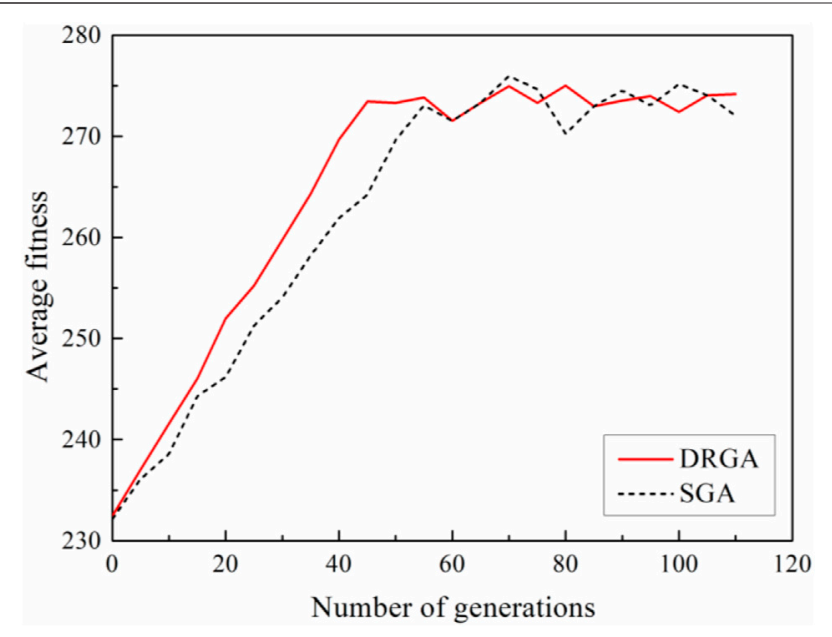

FIGURE 4 | Average fitness of every generation.

created in this method, and then an efficient GA iterative loop is established accordingly. The method searches a global optimal solution by loop calculating until the convergence criterion is satisfied. Two conditions can be adopted to terminate the calculation process. First, the difference between the results of two sequential calculations is small enough (e.g., less than 0.0001). Alternatively, enough loop iterations have been made (e.g., the iteration number exceeds 100).

\section{VALIDATION AND APPLICATION}

The improved adaptive immune system is built based on the improved adaptive methodology and the introduced immunity operator and distant relative pointer. The simulative aircraft wing box structure, which is an ideal spatial beam-slab structure system, is taken as a typical example to verify the proposed
DRGA method. The reliability-based structural optimization is calculated using the improved strategy as follows.

Figure 3 displays the finite element model (FEM) of a simulative aircraft wing box. The allowable stress is $66.89 \mathrm{kN} /$ $\mathrm{cm}^{2}$, and the coefficient of variation (Cov) for the element resistance is 0.05 . The elastic and shear modulus of the material are 6,689 and $2,300 \mathrm{kN} / \mathrm{cm}^{2}$, respectively. The density of the material is adopted as $2,700 \mathrm{~kg} / \mathrm{cm}^{3}$. All these above parameters are constant in the calculation process. In addition, the average of the external load is $44.45 \mathrm{kN}$, and the corresponding Cov is 0.2.

As shown in Figure 3, the FEM of the wing box consists of 10 beam elements and 13 slab elements. Let $A_{i}$ be the crosssectional area of the beam element, and it varies from 0.5 to $15 \mathrm{~cm}^{2}$. The thickness of the slab element is denoted by $B_{i}$, which ranges from 0.1 to $0.8 \mathrm{~cm}$. The resistance of components is independent to each other. The system reliability is limited by $\beta_{s}^{a}=3.5$. In the calculation process of system reliability optimization, $A_{i}$ and $B_{i}$ are adopted as the design variables, and the system mass is set as the objective function.

The RBSO question can be illustrated as follows:

$$
\left\{\begin{array}{l}
\min W=\sum_{i=1}^{10} A_{i} L_{i} \rho_{i}+\sum_{i=1}^{13} B_{i} M_{i} \rho_{i} \\
\text { s.t. } \quad 0.5 \leq A_{i} \leq 15, \quad 0.1 \leq B_{i} \leq 0.8, \quad \beta_{s} \geq 3.5
\end{array},\right.
$$

where $W$ is the structural mass, $L_{i}$ is the length of beam $i, M_{i}$ is the area of slab $i$, and $\rho$ is the density.

According to Eq. 11, $C$ can be determined as $910.4 \mathrm{~kg}$ when $A_{\mathrm{i}}$ and $B_{\mathrm{i}}$ equal the maximum values. Accordingly, the fitness function (Eq. 11) turns into the following:

$$
\begin{aligned}
F= & 910.4-\left(\sum_{i=1}^{10} A_{i} L_{i} \rho_{i}+\sum_{i=1}^{13} B_{i} M_{i} \rho_{i}\right) \\
& -\frac{1}{2 r}\left\{\max \left[0, \lambda+r\left(\beta_{s}-3.5\right)\right]^{2}-\lambda^{2}\right\} .
\end{aligned}
$$

Real number coding is adopted in the calculation, and the individual length is 23 . The population size is selected as 30 . The circle terminates when the genetic evolution generation runs up to 110 . The selection method adopts fitness proportion roulette. The optimization results of the wing box structure are summarized in Tables 1, 2, including the cross-sectional areas of beams and the thicknesses of slabs.

After optimization, the system reliability index is 3.5. The mass of the system is $302.47 \mathrm{~kg}$, while the original assumed mass of the structure $\left(A_{\mathrm{i}}=15 \mathrm{~cm}^{2} ; B_{\mathrm{i}}=0.8 \mathrm{~cm}\right)$ is $728.32 \mathrm{~kg}$. It indicates that the overall mass of the system is decreased by 
$425.85 \mathrm{~kg}$ via optimization, which ensures the system $58.5 \%$ lighter.

In order to analyze the performance of the proposed algorithm, the optimization statistical diagram obtained by the DRGA is compared with that obtained by the SGA, as shown in Figure 4. It is obvious that the average fitness is attained earlier based on the DRGA, which means a higher convergence efficiency.

\section{CONCLUSION}

This article mainly proposes an improved adaptive technology, termed as the distant relative genetic algorithm (DRGA), to enable the genetic algorithm (GA) to jump out of the local optimal solution, overcoming its associated precocious shortcoming and making a fast and stable convergence. A wing box structure is taken as the example to verify the proposed method. The results demonstrate that a more stable average fitness can be achieved earlier after introducing the distant relative control to the adaptive genetic algorithm, compared to the conventional standard genetic algorithm (SGA). Besides, the control of cross in the early genetic operations by the distant relative pointer can effectively avoid individual expansion, enabling the algorithm to jump out of local optimal solution and increase local search ability. A better global convergence and convergence efficiency have been achieved as a consequence.

\section{REFERENCES}

1. Qu CX, Li HN, Huo LS, and Yi TH. Optimum Value of Negative Stiffness and Additional Damping in the Civil Structures. J Struct Eng (2017) 143(8): 04017068. doi:10.1061/(asce)st.1943-541x.0001805

2. Qu CX, Yi TH, and Li HN. Mode Identification by Eigensystem Realization Algorithm through Virtual Frequency Response Function. Struct Control Health Monit (2019) 26(10):e2429. doi:10.1002/stc.2429

3. Cheng H, Li H-N, Yang YB, and Wang D-S. Seismic Fragility Analysis of Deteriorating RC Bridge Columns with Time-Variant Capacity index. Bull Earthquake Eng (2019) 17(7):4247-67. doi:10.1007/s10518-019-00628-x

4. Cheng $\mathrm{H}$, Wang DS, and $\mathrm{Li} \mathrm{HN}$. Investigation on Ultimate Lateral Displacements of Coastal Bridge Piers with Different Corrosion Levels along Height. J Bridge Eng (2021) 26(4):04021015. doi:10.1061/(asce) be.1943-5592.0001696

5. Sajedi S, and Huang Q. Reliability-based Life-Cycle-Cost Comparison of Different Corrosion Management Strategies. Eng Structures (2019) 186: 52-63. doi:10.1016/j.engstruct.2019.02.018

6. Mishra M, Keshavarzzadeh V, and Noshadravan A. Reliability-based Lifecycle Management for Corroding Pipelines. Struct Saf (2019) 76:1-14. doi:10.1016/ j.strusafe.2018.06.007

7. Santoro R, Muscolino G, and Elishakoff I. Optimization and Antioptimization Solution of Combined Parameterized and Improved Interval Analyses for Structures with Uncertainties. Comput Structures (2015) 149: 31-42. doi:10.1016/j.compstruc.2014.11.006

8. Holland JH. Adaptation in Natural Artificial Systems. Ann Arbor, MI: MIT Press (1975).

9. Goldberg DE. Computer-aided Gas Pipeline Operation Using Genetic Algorithms and Rule Learning. Ann Arbor, MI: Department of Civil Engineering, University of Michigan (1983).

\section{DATA AVAILABILITY STATEMENT}

The original contributions presented in the study are included in the article/supplementary material; further inquiries can be directed to the corresponding author.

\section{AUTHOR CONTRIBUTIONS}

Conceptualization: $\mathrm{HC}$ and X-CY. Methodology: X-CY. Software: $\mathrm{HC}$ and X-CY. Formal analysis: LF. Data curation: X-CY. Writing-original draft preparation: $\mathrm{HC}$ and $\mathrm{X}-\mathrm{CY}$. Writing-review and editing: HC and LF. Project administration: X-CY. Funding acquisition: HC. All authors have read and agreed to the published version of the manuscript.

\section{FUNDING}

This research is supported by the National Natural Science Foundation of China (Grant No. 52008186), the Natural Science Foundation of Jiangsu Province (Grant No. BK20200605), the Scientific Research Fund of Institute of Engineering Mechanics, China Earthquake Administration (Grant No. 2019D08), the Open Fund of State Key Laboratory of Coastal and Offshore Engineering, Dalian University of Technology (Grant No. LP2011), and the Fundamental Research Funds for the Central Universities (JUSRP12043). The authors greatly appreciate the financial supports.

10. Luo Y, and Kang Z. Layout Design of Reinforced Concrete Structures Using Two-Material Topology Optimization with Drucker-Prager Yield Constraints. Struct Multidisc Optim (2013) 47(1):95-110. doi:10.1007/s00158-012-0809-1

11. Barone G, and Frangopol DM. Life-cycle Maintenance of Deteriorating Structures by Multi-Objective Optimization Involving Reliability, Risk, Availability, Hazard and Cost. Struct Saf (2014) 48:40-50. doi:10.1016/ j.strusafe.2014.02.002

12. Srinivas M, and Patnaik LM. Adaptive Probabilities of Crossover and Mutation in Genetic Algorithms. IEEE Trans Syst Man Cybern (1994) 24(4):656-67. doi:10.1109/21.286385

13. Yan XC, An WG, and Chen WD. Reliability Analysis for Large-Scale Ship Structure. J Harbin Eng Univ (2004) 25(2):147-52. (in Chinese).

14. Thoft-Christensen P, and Murotsu Y. Application of Structural Systems Reliability Theory. Berlin: Spinger Press (1986). doi:10.1007/978-3-64282764-8

15. Wang GY. Theory of Soft Design in Engineering. Beijing: Science Press (1992). (in Chinese).

16. Yan XC, and Hua Y. Establishment of Genetic Algorithm Fitness Function in Reliability-Based Structural Optimization. Chin J Comput Mech (2009) 26(1): 120-3. (in Chinese)

Conflict of Interest: The authors declare that the research was conducted in the absence of any commercial or financial relationships that could be construed as a potential conflict of interest.

Copyright (c) 2021 Cheng, Yan and Fu. This is an open-access article distributed under the terms of the Creative Commons Attribution License (CC BY). The use, distribution or reproduction in other forums is permitted, provided the original author(s) and the copyright owner(s) are credited and that the original publication in this journal is cited, in accordance with accepted academic practice. No use, distribution or reproduction is permitted which does not comply with these terms. 